\title{
On Input-to-State Stability of Impulsive Stochastic Systems with Time Delays
}

\author{
Fengqi Yao, ${ }^{1}$ Pei Cheng, ${ }^{2}$ Hao Shen,, ${ }^{1}$ and Li Qiu ${ }^{3}$ \\ ${ }^{1}$ School of Electrical Engineering \& Information, Anhui University of Technology, Maanshan 243000, China \\ ${ }^{2}$ School of Mathematical Science, Anhui University, Hefei 230039, China \\ ${ }^{3}$ College of Mechatronics and Control Engineering, Shenzhen University, Shenzhen 518060, China
}

Correspondence should be addressed to Fengqi Yao; fqyao85@ahut.edu.cn

Received 6 January 2014; Accepted 1 March 2014; Published 3 April 2014

Academic Editor: Shuping He

Copyright (C) 2014 Fengqi Yao et al. This is an open access article distributed under the Creative Commons Attribution License, which permits unrestricted use, distribution, and reproduction in any medium, provided the original work is properly cited.

\begin{abstract}
This paper is concerned with $p$ th moment input-to-state stability ( $p$-ISS) and stochastic input-to-state stability (SISS) of impulsive stochastic systems with time delays. Razumikhin-type theorems ensuring $p$-ISS/SISS are established for the mentioned systems with external input affecting both the continuous and the discrete dynamics. It is shown that when the impulse-free delayed stochastic dynamics are $p$-ISS/SISS but the impulses are destabilizing, the $p$-ISS/SISS property of the impulsive stochastic systems can be preserved if the length of the impulsive interval is large enough. In particular, if the impulse-free delayed stochastic dynamics are $p$-ISS/SISS and the discrete dynamics are marginally stable for the zero input, the impulsive stochastic system is $p$-ISS/SISS regardless of how often or how seldom the impulses occur. To overcome the difficulties caused by the coexistence of time delays, impulses, and stochastic effects, Razumikhin techniques and piecewise continuous Lyapunov functions as well as stochastic analysis techniques are involved together. An example is provided to illustrate the effectiveness and advantages of our results.
\end{abstract}

\section{Introduction}

In practice, the performance of a real control system is affected more or less by uncertainties such as unmodeled dynamics, parameter perturbations, exogenous disturbances, and measurement errors [1]. To describe how solutions behave robustly to external inputs or disturbances, the concept of input-to-state stability (ISS) has been proven useful and effective in this regard. ISS was originally proposed by Sontag [2] for continuous-time systems. In view of its importance in the analysis and synthesis of nonlinear control systems [35], ISS and its variants such as local ISS, integral ISS, and exponential-weighted ISS have been investigated quite intensively and extended to different types of dynamical systems, for instance, discrete-time systems [6,7], switched systems $[1,8-11]$, network control systems [12], neural networks [1315], and so forth.

As it is well known, impulsive effect is likely to exist in a wide variety of evolutionary processes in which states are changed abruptly at certain moments of time in the fields such as medicine and biology, economics, electronics, and telecommunications [16]. Recently, Hespanha initiated the study of ISS for impulsive systems [17]. It was proved therein that impulsive systems possessing an exponential ISSLyapunov function are uniformly ISS over a certain class of impulse time sequences. Since time delay phenomena are often encountered in real world systems and the existence of time delay is a significant cause of instability and deteriorative performance, [18] investigated the ISS property for nonlinear impulsive systems with time delays by using Razumikhin techniques. And [19] was also concerned with ISS of impulsive systems with time delays, where ISS theorems different from those in [18] were established by adopting both Razumikhin techniques and Lyapunov-Krosovskii functional method.

In addition to the time delays and impulse effects, stochastic perturbations are always unavoidable in real systems (see [20-23] and references therein). Impulsive stochastic delayed systems incorporate impulses effects, stochastic perturbations, and time delays in one system simultaneously. During the last decade, there has been extensive interest in the study of force-free delayed impulsive stochastic systems; 
we refer to [24-28] and references therein. However, the corresponding theory for impulsive stochastic systems with external inputs has been relatively less developed.

The present paper aims to generalize the ISS results for deterministic delayed impulsive systems to stochastic settings. The $p$ th moment input-to-state stability ( $p$-ISS) and stochastic input-to-state stability (SISS) properties for impulsive stochastic delayed systems with external input affecting both the continuous dynamics and the impulses are investigated and Razumikhin-type theorems guaranteeing the $p$-ISS/SISS are established. The results indicate that when the delayed continuous stochastic dynamics are $p$-ISS/SISS and the discrete dynamics are destabilizing, the $p$-ISS/SISS properties of the original impulsive stochastic systems can be maintained if the length of impulsive interval is large enough. In particular, if the impulse-free delayed stochastic dynamics are $p$-ISS/SISS and the discrete dynamics are marginally stable for the zero input, the impulsive stochastic system is $p$ ISS/SISS regardless of how often or how seldom the impulses occur. As a byproduct, the criteria on pth moment global asymptotic stability ( $p$-GAS) and global asymptotical stability in probability (GASiP) are also derived. The initial idea of this paper came from the works for deterministic impulsive delayed systems [18] and impulse-free stochastic systems [1, 29], but its extension to impulsive stochastic delayed systems will be much more challenging due to the simultaneous existence of time delays, impulses, and stochastic effects.

The rest of this paper is organized as follows. In Section 2, some basic notations and definitions used throughout the paper are introduced. In Section 3, criteria ensuring uniform $p$-ISS/SISS/p-GAS/GASiP are established and applied to linear impulsive stochastic delayed systems. Section 4 provides a numerical example to illustrate the effectiveness and advantages of our results. Finally, Section 5 includes a summary and a discussion of some extensions of the paper.

\section{Preliminaries}

Throughout this paper, unless otherwise specified, we will employ the following notations. Let $\left.\left(\Omega, \mathscr{F}_{t}, \mathscr{F}_{t}\right\}_{t \geqslant 0}, \mathbb{P}\right)$ be a complete probability space with a filtration $\left\{\mathscr{F}_{t}\right\}_{t \geqslant 0}$ satisfying the usual conditions (i.e., it is right continuous and $\mathscr{F}_{0}$ contains all $\mathbb{P}$-null sets) and let $\mathbb{E}[\cdot]$ be the expectation operator with respect to the given probability measure $\mathbb{P}$. Let $w(t)=\left(w_{1}(t), \ldots, w_{d}(t)\right)^{\mathrm{T}}$ be a $d$-dimensional Brownian motion defined on the probability space. $\mathbb{R}=(-\infty,+\infty)$, $\mathbb{R}_{+}=[0,+\infty), \mathbb{N}=\{1,2,3, \ldots\}, \mathbb{R}^{n}$ denotes the $n$ dimensional real space equipped with the Euclidean norm $|\cdot|$, and $\mathbb{R}^{n \times m}$ denotes the $n \times m$-dimensional real matrix space.

Let $\tau \geqslant 0$ and $P C\left([-\tau, 0] ; \mathbb{R}^{n}\right)=\left\{\varphi:[-\tau, 0] \rightarrow \mathbb{R}^{n} \mid \varphi(t)\right.$ is continuous for all but at most a finite number of points $\bar{t}$, at which $\varphi\left(\bar{t}^{+}\right), \varphi\left(\bar{t}^{-}\right)$exist and $\left.\varphi\left(\bar{t}^{+}\right)=\varphi(\bar{t})\right\}$, where $\varphi\left(\bar{t}^{+}\right)$ and $\varphi\left(\bar{t}^{-}\right)$denote the right-hand and left-hand limits of $\varphi(t)$ at $\bar{t}$, respectively. We equip the linear space $P C\left([-\tau, 0] ; \mathbb{R}^{n}\right)$ with the norm $\|\varphi\|$ defined by $\|\varphi\|=\sup \{|\varphi(\theta)|:-\tau \leqslant \theta \leqslant$ $0\}$. Let $P C_{\mathscr{F}_{t}}^{b}\left([-\tau, 0] ; \mathbb{R}^{n}\right)$ be the family of all $\mathscr{F}_{t}$-measurable and bounded $P C\left([-\tau, 0] ; \mathbb{R}^{n}\right)$-valued random variables $\xi=$ $\{\xi(\theta):-\tau \leqslant \theta \leqslant 0\}$.
A function $\alpha: \mathbb{R}_{+} \rightarrow \mathbb{R}_{+}$is said to be of class $\mathscr{K}$ if it is continuous and strictly increasing and satisfies $\alpha(0)=0$; it is of class $\mathscr{K}_{\infty}$ if in addition $\alpha(s) \rightarrow \infty$ as $s \rightarrow \infty$. Note that if $\alpha$ is of class $\mathscr{K}_{\infty}$, then the inverse function $\alpha^{-1}$ is well defined and is also of class $\mathscr{K}_{\infty} \cdot v \mathscr{K}_{\infty}$ and $c \mathscr{K}_{\infty}$ are the subsets of $\mathscr{K}_{\infty}$ functions that are convex and concave, respectively. A function $\beta: \mathbb{R}_{+} \times \mathbb{R}_{+} \rightarrow \mathbb{R}_{+}$is said to be of class $\mathscr{K} \mathscr{L}$ if $\beta(\cdot, t) \in \mathscr{K}$ for each fixed $t \geqslant 0$ and $\beta(r, t)$ decreases to 0 as $t \rightarrow \infty$ for each fixed $r \geqslant 0$. The composition of two functions $\phi: A \rightarrow B$ and $\psi: B \rightarrow C$ is denoted by $\psi \circ \phi: A \rightarrow$ C.

If $A$ is a vector or a matrix, its transpose is denoted by $A^{\mathrm{T}}$. If $P$ is a square matrix, $P>0(P \leqslant 0)$ means that $P$ is a symmetric positive definite (negative semidefinite) matrix. $\underline{\lambda}(\cdot)$ and $\bar{\lambda}(\cdot)$ represent the minimum and maximum eigenvalues of the corresponding matrix, respectively, and $I$ stands for the identity matrix. The symbol $*$ is used in symmetric matrices to denote the entries which can be inferred by symmetry. Unless explicitly stated, all matrices are assumed to have real entries and compatible dimensions.

We consider the following impulsive stochastic nonlinear system with external inputs:

$$
\begin{array}{r}
\mathrm{d} x=f\left(t, x_{t}, u_{c}(t)\right) \mathrm{d} t+g\left(t, x_{t}, u_{c}(t)\right) \mathrm{d} w(t), \\
t \neq t_{k}, \quad t \geqslant t_{0}, \\
x\left(t_{k}\right)=I_{k}\left(t_{k}, x\left(t_{k}^{-}\right), u_{d}\left(t_{k}^{-}\right)\right), \quad k \in \mathbb{N},
\end{array}
$$

with initial data $x_{t_{0}}=\left\{x\left(t_{0}+\theta\right):-\tau \leqslant \theta \leqslant 0\right\}=\xi \epsilon$ $\mathbb{P} C_{\mathscr{F}_{t_{0}}}^{b}\left([-\tau, 0] ; \mathbb{R}^{n}\right)$, where $x \in \mathbb{R}^{n}$ and $x_{t}=\{x(t+\theta)$ : $-\tau \leqslant \theta \leqslant 0\}$ is regarded as a $P C\left([-\tau, 0] ; \mathbb{R}^{n}\right)$-valued random variable; $u_{c} \in \mathscr{L}_{\infty}^{m_{1}}$ is locally essentially bounded external input and $u_{d} \in \mathscr{L}_{\mathrm{o}}^{m_{2}}$ is the impulsive disturbance input; $\mathscr{L}_{\mathrm{o}}^{m}$ denotes the set of all locally essentially bounded function $u: \mathbb{R}_{+} \rightarrow \mathbb{R}^{m}$ with norm $\|u\|_{\infty}=\operatorname{ess~sup~}_{t \geqslant t_{0}}|u(t)| ;\|u\|_{[a, b]}=$ ess $\sup _{t \in[a, b]}|u(t)|$; both $f:\left[t_{0}, \infty\right) \times \mathbb{R}^{n} \times \mathbb{R}^{m_{1}} \rightarrow \mathbb{R}^{n}$ and $g$ : $\left[t_{0}, \infty\right) \times \mathbb{R}^{n} \times \mathbb{R}^{m_{1}} \rightarrow \mathbb{R}^{n \times d}$ are uniformly locally Lipschitz with respect to $x$ and $u ; I_{k}:\left[t_{0}, \infty\right) \times \mathbb{R}^{n} \times \mathbb{R}^{m_{2}} \rightarrow \mathbb{R}^{n}$ represents the impulsive perturbation of $x$ at $t_{k}$ and satisfies $\left|I_{k}\left(t_{k}, x, u\right)\right|<\infty ;\left\{t_{k}\right\}_{k \in \mathbb{N}}$ is a strictly increasing sequence of impulse times. We use $\mathcal{S}_{\min }(\beta)$ and $\mathcal{S}_{\text {all }}$ to denote the class of impulsive time sequences that satisfy inf $\operatorname{in}_{k \in \mathbb{N}}\left\{t_{k}-t_{k-1}\right\} \geqslant \beta$ and the set containing all impulse time sequences, respectively.

Moreover, we assume that $f(t, 0,0)=g(t, 0,0)=I_{k}(t, 0$, $0) \equiv 0$ for all $t \geqslant t_{0}, k \in \mathbb{N}$; then system (1) admits a trivial solution $x(t) \equiv 0$. The input pair $\left(u_{c}, u_{d}\right)$ is said to be admissible, denoted by $\left(u_{c}, u_{d}\right) \in \mathscr{U}$, if $u_{c} \in \mathscr{L}_{\infty}^{m_{1}}, u_{d} \in \mathscr{L}_{\infty}^{m_{2}}$ guarantee the the existence of a unique solution to system (1).

On the foundation of the ISS concepts for impulse-free stochastic systems $[1,29,30]$ and those for deterministic impulsive systems [18], we proposed the following definitions for impulsive stochastic delayed systems (1).

Definition 1. For a prescribed sequence $\left\{t_{k}\right\}_{k \in \mathbb{N}}$, system (1) is said to be $p$ th $(p>0)$ moment input-to-state stable (ISS) if there exist functions $\beta \in \mathscr{K} \mathscr{L}, \alpha, \gamma_{c}, \gamma_{d} \in \mathscr{K}_{\infty}$ such that, 
for every initial condition $\xi \in P C_{\mathscr{F}_{t_{0}}}^{b}$ and every input pair $\left(u_{c}, u_{d}\right) \in \mathscr{U}$,

$$
\begin{aligned}
\alpha\left(\mathbb{E}|x(t)|^{p}\right) \leqslant & \beta\left(\mathbb{E}\|\xi\|^{p}, t-t_{0}\right)+\gamma_{c}\left(\left\|u_{c}\right\|_{\left[t_{0}, t\right]}\right) \\
& +\gamma_{d}\left(\max _{t_{k} \in\left(t_{0}, t\right]}\left\{\left|u_{d}\left(t_{k}^{-}\right)\right|\right\}\right), \quad t \geqslant t_{0} .
\end{aligned}
$$

Definition 2. For a given sequence $\left\{t_{k}\right\}_{k \in \mathbb{N}}$, system (1) is said to be stochastic input-to-state stable (SISS), if, for any $\varepsilon>0$, there exist functions $\beta \in \mathscr{K} \mathscr{L}$ and $\alpha, \gamma_{c}, \gamma_{d} \in \mathscr{K}_{\infty}$, such that, for every initial condition $\xi \in P C_{\mathscr{F}_{t_{0}}}^{b}$ and every input pair $\left(u_{c}, u_{d}\right) \in \mathscr{U}$,

$$
\begin{aligned}
& P\left\{\alpha(|x(t)|)<\beta\left(\|\xi\|, t-t_{0}\right)+\gamma_{c}\left(\left\|u_{c}\right\|_{\left[t_{0}, t\right]}\right)\right. \\
& \left.\quad+\gamma_{d}\left(\max _{t_{k} \in\left(t_{0}, t\right]}\left\{\left|u_{d}\left(t_{k}^{-}\right)\right|\right\}\right)\right\}>1-\varepsilon, \quad t \geqslant t_{0} .
\end{aligned}
$$

Remark 3. Redefining $\beta$ and $\gamma_{c}, \gamma_{d}$, one can assume that $\alpha$ in (2) or (3) is the identity: if $\alpha(r) \leqslant \beta(s, t)+\gamma_{c}(v)+\gamma_{d}(\nu)$ holds, then also $r \leqslant \alpha^{-1}\left(\beta(s, t)+\gamma_{c}(v)+\gamma_{d}(\nu)\right) \leqslant \alpha^{-1}(3 \beta(s, t))+$ $\alpha^{-1}\left(3 \gamma_{c}(v)\right)+\alpha^{-1}\left(3 \gamma_{d}(\nu)\right)$. We know by Lemma 4.2 in [31] that $\alpha^{-1}(3 \beta(\cdot, \cdot)) \in \mathscr{K} \mathscr{L}$ and $\alpha^{-1}\left(3 \gamma_{c}(\cdot)\right), \alpha^{-1}\left(3 \gamma_{d}(\cdot)\right) \in \mathscr{K}_{\infty}$. So estimates of the same type as (2) and (3) but with no " $\alpha$ " are obtained.

In the following, we will define $p$-GAS and GASiP in the form of $\mathscr{K} \mathscr{L}$ function, which present very close analogy to $p$-ISS and SISS, respectively.

Definition 4. For a prescribed sequence $\left\{t_{k}\right\}_{k \in \mathbb{N}}$, system (1) with input $u_{c} \equiv 0, u_{d} \equiv 0$ is said to be $p$ th $(p>0)$ moment globally asymptotically stable (GAS) if there exists a function $\beta \in \mathscr{K} \mathscr{L}$ such that, for every initial condition $\xi \in P C_{\mathscr{F}_{t_{0}}}^{b}$,

$$
\mathbb{E}|x(t)|^{p} \leqslant \beta\left(\mathbb{E}\|\xi\|^{p}, t-t_{0}\right), \quad t \geqslant t_{0} .
$$

Definition 5. For a given sequence $\left\{t_{k}\right\}_{k \in \mathbb{N}}$, system (1) with input $u_{c} \equiv 0, u_{d} \equiv 0$ is said to be globally asymptotically stable in probability (GASiP), if, for any $\varepsilon>0$, there exists a function $\beta \in \mathscr{K} \mathscr{L}$, such that, for every initial condition $\xi \in P C_{\mathscr{F}_{t_{0}}}^{b}$,

$$
P\left\{|x(t)|<\beta\left(\|\xi\|, t-t_{0}\right)\right\}>1-\varepsilon, \quad t \geqslant t_{0} .
$$

Remark 6. By the vanishing of $\gamma_{c}(s)$ and $\gamma_{d}(s)$ at $s=0$, (2) and (3) will degenerate to (4) and (5), respectively, when $u \equiv 0$, which means that $p$-ISS/SISS of system (1) implies $p$ GAS/GASiP of the corresponding unforced system.

System (1) is said to be uniformly $p$-ISS or uniformly SISS over a given class of admissible impulsive time sequences $\delta$ if (2) or (3) holds for every sequence in $\delta$ with functions $\alpha, \beta$, $\gamma_{c}$, and $\gamma_{d}$ independent of the choice of the sequence. Uniform $p$-GAS and uniform GASiP can be defined similarly.
Definition 7 (see [24]). A function $V: \mathbb{R}_{+} \times \mathbb{R}^{n} \rightarrow \mathbb{R}_{+}$is said to be of class $v_{0}$ if the following hold true.

(i) $V$ is continuous on each of the sets $\left[t_{k-1}, t_{k}\right) \times \mathbb{R}^{n}$ and for each $x, y \in \mathbb{R}^{n}, t \in\left[t_{k-1}, t_{k}\right), k \in \mathbb{N}$, and $\lim _{(t, y) \rightarrow\left(t_{k}^{-}, x\right)} V(t, y)=V\left(t_{k}^{-}, x\right)$ exists.

(ii) $V(t, x)$ is once continuously differentiable in $t$ and twice in $x$ in each of the sets $\left(t_{k-1}, t_{k}\right) \times \mathbb{R}^{n}, k \in \mathbb{N}$.

If $V \in v_{0}$, define an operator $\mathscr{L} V$ [24] with respect to system (1) by

$$
\begin{aligned}
\mathscr{L} V(t, \varphi, u)= & V_{t}(t, \varphi(0))+V_{x}(t, x) f(t, \varphi, u) \\
& +\frac{1}{2} \operatorname{trace}\left[g^{\mathrm{T}}(t, \varphi, u) V_{x x}(t, x) g(t, \varphi, u)\right],
\end{aligned}
$$

where

$$
\begin{aligned}
& V_{t}(t, x)=\frac{\partial V(t, x)}{\partial t}, \\
& V_{x}(t, x)=\left(\frac{\partial V(t, x)}{\partial x_{1}}, \ldots, \frac{\partial V(t, x)}{\partial x_{n}}\right), \\
& V_{x x}(t, x)=\left(\frac{\partial^{2} V(t, x)}{\partial x_{i} \partial x_{j}}\right)_{n \times n} .
\end{aligned}
$$

\section{Main Results}

In this section, we will develop Lyapunov-Razumikhin methods and establish some criteria which provide sufficient conditions for the $p$-ISS and SISS properties of impulsive stochastic delayed systems (1).

Theorem 8. Assume that there exist functions $V \in v_{0}, \chi_{1}, \chi_{2} \in$ $\mathscr{K}_{\infty}, \alpha_{1} \in c \mathscr{K}_{\infty}, \alpha_{2} \in v \mathscr{K}_{\infty}$ and scalars $q>1, c>0, \mu \in$ $[1, q)$ such that

(i) $\alpha_{1}\left(|x|^{p}\right) \leqslant V(t, x) \leqslant \alpha_{2}\left(|x|^{p}\right)$;

(ii) $\mathbb{E} \mathscr{L} V(t, \varphi) \leqslant-c \mathbb{E} V(t, \varphi(0))+\chi_{1}\left(\left|u_{c}(t)\right|\right)$, for all $t \geqslant$ $t_{0}, t \neq t_{k}$ and $\varphi \in P C_{\mathscr{F}_{t}}\left([-\tau, 0] ; \mathbb{R}^{n}\right)$ whenever $\mathbb{E} V(t+$ $\theta, \varphi(\theta)) \leqslant q \mathbb{E} V(t, \varphi(0))$;

(iii) $\mathbb{E} V\left(t_{k}, I_{k}\left(t_{k}^{-}, x, u_{d}\right)\right) \leqslant \mu \mathbb{E} V\left(t_{k}, x\right)+\chi_{2}\left(\left|u_{d}\right|\right)$.

Then for any given $\rho>0$ satisfying $\mu \mathrm{e}^{-c \rho}<1$, system (1) is uniformly $p$-ISS over $\mathcal{S}_{\min }(\rho)$. In particular, when $\mu=1$, system (1) is uniformly p-ISS over $\mathcal{S}_{\text {all }}$.

Proof. Since $\mu \mathrm{e}^{-c \rho}<1$, then $0<1+\mathrm{e}^{-c \rho}-1 / \mu<1$ and there exists $c^{\prime}>0$ such that $c \max \left\{\mu \mathrm{e}^{-c \rho}, 1+\mathrm{e}^{-c \rho}-1 / \mu\right\}<c^{\prime}<c$. We choose $\lambda \in\left(0, c^{\prime}\right)$ such that $(q / \mu) \mathrm{e}^{-\lambda \tau}>1, \mu \mathrm{e}^{-(c-\lambda) \rho} \leqslant 1$, $\lambda \leqslant c-\mu\left(c-c^{\prime}\right)$. Let $\left\{t_{k}\right\}_{k \in \mathbb{N}}$ be any impulsive time sequence belonging to $\mathcal{S}_{\min }(\rho)$. For simplicity, we write $V(t, x)=V(t)$. Define

$$
J(t)=\mathrm{e}^{\lambda\left(t-t_{0}\right)}\left[\mathbb{E} V(t)-J_{0}(t)\right], \quad t \geqslant t_{0}-\tau,
$$


where $J_{0}(t)=\left(1 /\left(c-c^{\prime}\right)\right) \chi_{1}\left(\left\|u_{c}\right\|_{\left[t_{0}, t\right]}\right)+$ $\sum_{t_{k} \in\left(t_{0}, t\right]} \mathrm{e}^{-\lambda\left(t-t_{k}\right)} \chi_{2}\left(\left|u_{d}\left(t_{k}^{-}\right)\right|\right)$for $t \geqslant t_{0}$ and $J_{0}(t)=0$ for $t \in\left[t_{0}-\tau, t_{0}\right]$. We claim that

$$
J(t) \leqslant \alpha_{2}\left(\mathbb{E}\|\xi\|^{p}\right), \quad t \geqslant t_{0} .
$$

We first prove that (9) holds for $t \in\left[t_{0}, t_{1}\right)$. By condition (i) and Jensen's inequality, it is easy to see that

$$
\begin{aligned}
J(t) & =\mathbb{E} V(t) \mathrm{e}^{\lambda\left(t-t_{0}\right)} \\
& \leqslant \alpha_{2}\left(\mathbb{E}|x(t)|^{p}\right) \\
& \leqslant \alpha_{2}\left(\mathbb{E}\|\xi\|^{p}\right), \quad t \in\left[t_{0}-\tau, t_{0}\right] .
\end{aligned}
$$

If (9) is not true for $t \in\left[t_{0}, t_{1}\right)$, there must exist some $t \in$ $\left[t_{0}, t_{1}\right)$ such that $J(t)>\alpha_{2}\left(\mathbb{E}\|\xi\|^{p}\right)$. Let $t^{*}=\inf \left\{t \in\left[t_{0}, t_{1}\right)\right.$ : $\left.J(t)>\alpha_{2}\left(\mathbb{E}\|\xi\|^{p}\right)\right\}$. Then by the right continuity of $J(t)$ in $t \in$ $\left[t_{0}, t_{1}\right)$ and noticing $(10)$, we have $t^{*} \geqslant t_{0}$ and

$$
\begin{array}{r}
J\left(t^{*}\right)=\alpha_{2}\left(\mathbb{E}\|\xi\|^{p}\right), \quad J(t) \leqslant \alpha_{2}\left(\mathbb{E}\|\xi\|^{p}\right), \\
t \in\left[t_{0}-\tau, t^{*}\right), \quad D^{+} J\left(t^{*}\right)>0 .
\end{array}
$$

Because $J\left(t^{*}\right) \geqslant J\left(t^{*}+s\right), s \in[-\tau, 0]$ implies

$$
\begin{aligned}
\mathbb{E} V\left(t^{*}\right) & \geqslant \mathrm{e}^{\lambda s} \mathbb{E} V\left(t^{*}+s\right)-\mathrm{e}^{\lambda s} J_{0}\left(t^{*}+s\right)+J_{0}\left(t^{*}\right) \\
& \geqslant \mathrm{e}^{-\lambda \tau} \mathbb{E} V\left(t^{*}+s\right)>\frac{\mu}{q} \mathbb{E} V\left(t^{*}+s\right) \\
& \geqslant \frac{1}{q} \mathbb{E} V\left(t^{*}+s\right), \quad s \in[-\tau, 0],
\end{aligned}
$$

it follows from condition (ii) that

$$
\mathbb{E} \mathscr{L} V\left(t^{*}\right) \leqslant-c \mathbb{E} V\left(t^{*}\right)+\chi_{1}\left(\left|u_{c}\left(t^{*}\right)\right|\right) .
$$

For $\rho>0$ sufficiently small satisfying $t^{*}+\rho<t_{1}$, by the Itô formula, we have

$$
\mathbb{E} V\left(t^{*}+\rho\right)-\mathbb{E} V\left(t^{*}\right)=\int_{t^{*}}^{t^{*}+\rho} \mathbb{E} \mathscr{L} V\left(s, x_{s}\right) \mathrm{d} s
$$

which yields

$$
\limsup _{\rho \rightarrow 0^{+}} \frac{\mathbb{E} V\left(t^{*}+\rho\right)-\mathbb{E} V\left(t^{*}\right)}{\rho}=\limsup _{\rho \rightarrow 0^{+}} \frac{1}{\rho} \int_{t^{*}}^{t^{*}+\rho} \mathbb{E} \mathscr{L} V(s) \mathrm{d} s ;
$$

that is,

$$
D^{+} \mathbb{E} V\left(t^{*}\right)=\mathbb{E} \mathscr{L} V\left(t^{*}\right) \leqslant-c \mathbb{E} V\left(t^{*}\right)+\chi_{1}\left(\left|u_{c}\left(t^{*}\right)\right|\right),
$$

where $D^{+} \mathbb{E} V(t) \triangleq \lim _{\sup } \rightarrow 0^{+}[\mathbb{E} V(t+\rho)-\mathbb{E} V(t)] / \rho$. On the other hand, $J\left(t^{*}\right)=\alpha_{2}\left(\mathbb{E}\|\xi\|^{p}\right) \geqslant 0$ implies

$$
\mathbb{E} V\left(t^{*}\right) \geqslant J_{0}\left(t^{*}\right) \text {. }
$$

Therefore, from (16) and (17), and noticing $J_{0}(t)=(1 /(c-$ $\left.\left.c^{\prime}\right)\right) \chi_{1}\left(\left\|u_{c}\right\|_{\left[t_{0}, t\right]}\right)$ and $D^{+} J_{0}(t) \geqslant 0$ for $t \in\left[t_{0}, t_{1}\right)$, we have

$$
\begin{gathered}
D^{+} J\left(t^{*}\right)=\mathrm{e}^{\lambda\left(t^{*}-t_{0}\right)}\left[D^{+} \mathbb{E} V\left(t^{*}\right)+\lambda \mathbb{E} V\left(t^{*}\right)\right. \\
\left.-\lambda J_{0}\left(t^{*}\right)-D^{+} J_{0}\left(t^{*}\right)\right] \\
\leqslant \mathrm{e}^{\lambda\left(t^{*}-t_{0}\right)}\left[-(c-\lambda) \mathbb{E} V\left(t^{*}\right)+\chi_{1}\left(\left|u_{c}\left(t^{*}\right)\right|\right)\right. \\
\left.\quad-\lambda J_{0}\left(t^{*}\right)\right] \\
\leqslant \mathrm{e}^{\lambda\left(t^{*}-t_{0}\right)}\left[-c J_{0}\left(t^{*}\right)+\chi_{1}\left(\left|u_{c}\left(t^{*}\right)\right|\right)\right] \\
\leqslant \frac{-c^{\prime}}{c-c^{\prime}} \mathrm{e}^{\lambda\left(t^{*}-t_{0}\right)} \chi_{1}\left(\left\|u_{c}\right\|_{\left[t_{0}, t^{*}\right]}\right)<0,
\end{gathered}
$$

which contradicts $D^{+} J\left(t^{*}\right)>0$. Therefore, (9) holds for $t \in$ $\left[t_{0}-\tau, t_{1}\right)$.

Suppose that (9) holds for $t \in\left[t_{0}-\tau, t_{m}\right)$, where $m \geqslant 1$, $m \in \mathbb{N}$. We will prove that (9) holds for $t \in\left[t_{m}, t_{m+1}\right)$. To this end, we claim that

$$
J_{1}\left(t_{m}^{-}\right) \leqslant \alpha_{2}\left(\mathbb{E}\|\xi\|^{p}\right)
$$

where $J_{1}(t)=\mathrm{e}^{\lambda\left(t-t_{0}\right)}\left[\mu \mathbb{E} V(t)-J_{0}(t)\right]$. If not, then $J_{1}\left(t_{m}^{-}\right)>$ $\alpha_{2}\left(\mathbb{E}\|\xi\|^{p}\right)$. We consider the following two cases.

Case 1. $J_{1}(t)>\alpha_{2}\left(\mathbb{E}\|\xi\|^{p}\right)$ for all $t \in\left[t_{m-1}, t_{m}\right)$. It is easy to see that $J_{1}(t)>\alpha_{2}\left(\mathbb{E}\|\xi\|^{p}\right) \geqslant J(t+\theta)$ for $t \in\left[t_{m-1}, t_{m}\right)$ and $\theta \in[-\tau, 0]$. It follows that

$$
\begin{aligned}
\mathbb{E} V(t+\theta) & <\mathrm{e}^{-\lambda \theta}\left[\mu \mathbb{E} V(t)-J_{0}(t)\right]+J_{0}(t+\theta) \\
& \leqslant \mathrm{e}^{\lambda \tau} \mu \mathbb{E} V(t)-\mathrm{e}^{-\lambda \theta} J_{0}(t)+J_{0}(t+\theta) \\
& <q \mathbb{E} V(t), \quad t \in\left[t_{m-1}, t_{m}\right), \theta \in[-\tau, 0] .
\end{aligned}
$$

The last inequality comes from the fact that $(q / \mu) \mathrm{e}^{-\lambda \tau}>1$, and

$$
\begin{aligned}
J_{0}(t+\theta)= & \frac{1}{c-c^{\prime}} \chi_{1}\left(\left\|u_{c}\right\|_{\left[t_{0}, t+\theta\right]}\right) \\
& +\sum_{t_{k} \in\left(t_{0}, t+\theta\right]} \mathrm{e}^{-\lambda\left(t+\theta-t_{k}\right)} \chi_{2}\left(\left|u_{d}\left(t_{k}^{-}\right)\right|\right) \\
\leqslant & \frac{\mathrm{e}^{-\lambda \theta}}{c-c^{\prime}} \chi_{1}\left(\left\|u_{c}\right\|_{\left[t_{0}, t\right]}\right) \\
& +\sum_{t_{k} \in\left(t_{0}, t\right]} \mathrm{e}^{-\lambda\left(t+\theta-t_{k}\right)} \chi_{2}\left(\left|u_{d}\left(t_{k}^{-}\right)\right|\right) \\
= & \mathrm{e}^{-\lambda \theta} J_{0}(t) .
\end{aligned}
$$

By condition (ii), (20) indicates that

$$
\mathbb{E} \mathscr{L} V(t) \leqslant-c \mathbb{E} V(t)+\chi_{1}\left(\left|u_{c}(t)\right|\right), \quad t \in\left[t_{m-1}, t_{m}\right) .
$$


By Itô's formula, we have

$$
\begin{aligned}
\mathrm{e}^{c t_{m}} \mathbb{E} V\left(t_{m}^{-}\right)= & \mathrm{e}^{c t_{m-1}} \mathbb{E} V\left(t_{m-1}\right) \\
& +\int_{t_{m-1}}^{t_{m}} \mathrm{e}^{c s}[c \mathbb{E} V(s)+\mathbb{E} \mathscr{L} V(s)] \mathrm{d} s \\
\leqslant & \mathrm{e}^{c t_{m-1}} \mathbb{E} V\left(t_{m-1}\right) \\
& +\int_{t_{m-1}}^{t_{m}} \mathrm{e}^{c s} \chi_{1}\left(\left|u_{c}(s)\right|\right) \mathrm{d} s \\
\leqslant & \mathrm{e}^{c t_{m-1} \mathbb{E} V\left(t_{m-1}\right)} \\
& +\frac{1}{c} \mathrm{e}^{c t_{m}} \chi_{1}\left(\left\|u_{c}\right\|_{\left[t_{0}, t_{m}\right)}\right) ;
\end{aligned}
$$

thus,

$$
\mathbb{E} V\left(t_{m}^{-}\right) \leqslant \mathrm{e}^{-c\left(t_{m}-t_{m-1}\right)} \mathbb{E} V\left(t_{m-1}\right)+\frac{1}{c} \chi_{1}\left(\left\|u_{c}\right\|_{\left[t_{0}, t_{m}\right)}\right) .
$$

On the other hand, $J\left(t_{m-1}\right) \leqslant \alpha_{2}\left(\mathbb{E}\|\xi\|^{p}\right)$ implies

$$
\begin{aligned}
\mathbb{E} V\left(t_{m-1}\right) \leqslant & \mathrm{e}^{-\lambda\left(t_{m-1}-t_{0}\right)} \alpha_{2}\left(\mathbb{E}\|\xi\|^{p}\right)+J_{0}\left(t_{m-1}\right) \\
\leqslant & \mathrm{e}^{-\lambda\left(t_{m-1}-t_{0}\right)} \alpha_{2}\left(\mathbb{E}\|\xi\|^{p}\right) \\
& +\frac{1}{c-c^{\prime}} \chi_{1}\left(\left\|u_{c}\right\|_{\left[t_{0}, t_{m-1}\right]}\right) \\
& +\sum_{k=1}^{m-1} \mathrm{e}^{-\lambda\left(t_{m-1}-t_{k}\right)} \chi_{2}\left(\left|u_{d}\left(t_{k}^{-}\right)\right|\right) .
\end{aligned}
$$

Substituting (25) into (24) and noticing the fact that inf $\left\{t_{k}-\right.$ $\left.t_{k-1}\right\} \geqslant \rho$, we have

$$
\begin{aligned}
\mathbb{E} V\left(t_{m}^{-}\right) \leqslant & \mathrm{e}^{-c\left(t_{m}-t_{m-1}\right)-\lambda\left(t_{m-1}-t_{0}\right)} \alpha_{2}\left(\|\xi\|^{p}\right) \\
& +\left(\frac{1}{c}+\frac{\mathrm{e}^{-c \rho}}{c-c^{\prime}}\right) \chi_{1}\left(\left\|u_{c}\right\|_{\left[t_{0}, t_{m}\right)}\right) \\
& +\mathrm{e}^{-c\left(t_{m}-t_{m-1}\right)} \sum_{k=1}^{m-1} \mathrm{e}^{-\lambda\left(t_{m-1}-t_{k}\right)} \chi_{2}\left(\left|u_{d}\left(t_{k}^{-}\right)\right|\right) .
\end{aligned}
$$

Substituting (26) into $J_{1}\left(t_{m}^{-}\right)$yields

$$
\begin{aligned}
& J_{1}\left(t_{m}^{-}\right)=\mathrm{e}^{\lambda\left(t_{m}-t_{0}\right)}[\left.\mu \mathbb{E} V\left(t_{m}^{-}\right)-J_{0}\left(t_{m}^{-}\right)\right] \\
& \leqslant \mu \mathrm{e}^{\lambda\left(t_{m}-t_{0}\right)}\left[\mathrm{e}^{-c\left(t_{m}-t_{m-1}\right)-\lambda\left(t_{m-1}-t_{0}\right)} \alpha_{2}\left(\mathbb{E}\|\xi\|^{p}\right)\right. \\
&+\left(\frac{\mathrm{e}^{-c \rho}}{c-c^{\prime}}+\frac{1}{c}\right) \chi_{1}\left(\left\|u_{c}\right\|_{\left[t_{0}, t_{m}\right)}\right) \\
&+\mathrm{e}^{-c\left(t_{m}-t_{m-1}\right)} \sum_{k=1}^{m-1} \mathrm{e}^{-\lambda\left(t_{m-1}-t_{k}\right)} \\
&\left.\times \chi_{2}\left(\left|u_{d}\left(t_{k}^{-}\right)\right|\right)\right]
\end{aligned}
$$

$$
\begin{aligned}
& -\mathrm{e}^{\lambda\left(t_{m}-t_{0}\right)}\left[\frac{1}{c-c^{\prime}} \chi_{1}\left(\left\|u_{c}\right\|_{\left[t_{0}, t_{m}\right)}\right)\right. \\
& \left.+\sum_{k=1}^{m-1} \mathrm{e}^{-\lambda\left(t_{m}-t_{k}\right)} \chi_{2}\left(\left|u_{d}\left(t_{k}^{-}\right)\right|\right)\right] \\
& \leqslant \mu \mathrm{e}^{-(c-\lambda) \rho} \alpha_{2}\left(\mathbb{E}\|\xi\|^{p}\right) \\
& +\mathrm{e}^{\lambda\left(t_{m}-t_{0}\right)}\left[\mu\left(\frac{\mathrm{e}^{-c \rho}}{c-c^{\prime}}+\frac{1}{c}\right)-\frac{1}{c-c^{\prime}}\right] \chi_{1}\left(\left\|u_{c}\right\|_{\left[t_{0}, t_{m}\right)}\right) \\
& +\left(\mu \mathrm{e}^{-(c-\lambda) \rho}-1\right) \sum_{k=1}^{m-1} \mathrm{e}^{\lambda\left(t_{k}-t_{0}\right)} \chi_{2}\left(\left|u_{d}\left(t_{k}^{-}\right)\right|\right) \\
& \leqslant
\end{aligned}
$$

The last inequality holds because $\mu \mathrm{e}^{-(c-\lambda) \rho} \leqslant 1$ and $\mu\left(\left(\left(\mathrm{e}^{-c \rho}\right) /\left(c-c^{\prime}\right)\right)+(1 / c)\right)-\left(1 /\left(c-c^{\prime}\right)\right) \leqslant 0$. This is a contradiction.

Case 2. There exists some $t \in\left[t_{m-1}, t_{m}\right)$ such that $J_{1}(t) \leqslant$ $\alpha_{2}\left(\mathbb{E}\|\xi\|^{p}\right)$. Set $t^{\prime}=\sup \left\{t \in\left[t_{m-1}, t_{m}\right): J_{1}(t) \leqslant \alpha_{2}\left(\mathbb{E}\|\xi\|^{p}\right)\right\}$. Then $J_{1}\left(t^{\prime}\right)=\alpha_{2}\left(\mathbb{E}\|\xi\|^{p}\right)$ and $J_{1}(t)>\alpha_{2}\left(\mathbb{E}\|\xi\|^{p}\right)$ for $t \in\left(t^{\prime}, t_{m}\right)$. Thus, for $t \in\left[t^{\prime}, t_{m}\right), J_{1}(t) \geqslant \alpha_{2}\left(\mathbb{E}\|\xi\|^{p}\right) \geqslant J(t+\theta)$ for $\theta \in[-\tau, 0]$. This implies that (20) holds for $\theta \in[-\tau, 0]$, $t \in\left[t^{\prime}, t_{m}\right)$. Thus, by condition (ii),

$$
\begin{array}{r}
D^{+} \mathbb{E} V(t)=\mathbb{E} \mathscr{L} V(t) \leqslant-c \mathbb{E} V(t)+\chi_{1}\left(\left|u_{c}(t)\right|\right), \\
t \in\left[t^{\prime}, t_{m}\right) .
\end{array}
$$

Hence, noticing the fact that $J_{0}(t) \geqslant 0, D^{+} J_{0}(t) \geqslant 0$ for $t \in$ $\left[t^{\prime}, t_{m}\right)$, we have

$$
\begin{aligned}
& D^{+} J_{1}(t)=\mathrm{e}^{\lambda\left(t-t_{0}\right)}\left[\lambda \mu \mathbb{E} V(t)-\lambda J_{0}(t)+\mu D^{+} \mathbb{E} V(t)\right.\left.-D^{+} J_{0}(t)\right] \\
& \leqslant \mathrm{e}^{\lambda\left(t-t_{0}\right)}[-\mu(c-\lambda) \mathbb{E} V(t)-\lambda J_{0}(t) \\
&\left.+\mu \chi_{1}\left(\left|u_{c}(t)\right|\right)-D^{+} J_{0}(t)\right] \\
& \leqslant \mu \mathrm{e}^{\lambda\left(t-t_{0}\right)}\left[-(c-\lambda) \mathbb{E} V(t)+\chi_{1}\left(\left|u_{c}(t)\right|\right)\right], \\
& t \in\left[t^{\prime}, t_{m}\right) .
\end{aligned}
$$

Because $J_{1}(t) \geqslant \alpha_{2}\left(\mathbb{E}\|\xi\|^{p}\right)>0$ for $t \in\left[t^{\prime}, t_{m}\right)$, there holds $\mathbb{E} V(t)>(1 / \mu) J_{0}(t)>\left(1 / \mu\left(c-c^{\prime}\right)\right) \chi_{1}\left(\left\|u_{c}\right\|_{\left[t_{0}, t\right]}\right)$ for $t \in\left[t^{\prime}, t_{m}\right)$. Substituting this inequality with (29), and recalling the choice of $\lambda$, it follows that

$$
D^{+} J_{1}(t) \leqslant-\mathrm{e}^{\lambda\left(t-t_{0}\right)}\left(\frac{c-\lambda}{c-c^{\prime}}-\mu\right) \chi_{1}\left(\left\|u_{c}\right\|_{\infty}\right) \leqslant 0,
$$

which yields the following contradiction: $\alpha_{2}\left(\mathbb{E}\|\xi\|^{p}\right)<$ $J_{1}\left(t_{m}^{-}\right) \leqslant J_{1}\left(t^{\prime}\right)=\alpha_{2}\left(\mathbb{E}\|\xi\|^{p}\right)$.

Therefore, we have $J_{1}\left(t_{m}^{-}\right) \leqslant \alpha_{2}\left(\mathbb{E}\|\xi\|^{p}\right)$. Using condition (iii), we obtain that $J\left(t_{m}\right) \leqslant J_{1}\left(t_{m}^{-}\right) \leqslant \alpha_{2}\left(\mathbb{E}\|\xi\|^{p}\right)$. Repeating 
the argument used in the proof of $J(t) \leqslant \alpha_{2}\left(\mathbb{E}\|\xi\|^{p}\right)$ for $t \in$ $\left[t_{0}, t_{1}\right)$, we can get $J(t) \leqslant \alpha_{2}\left(\mathbb{E}\|\xi\|^{p}\right)$ for $t \in\left[t_{m}, t_{m+1}\right)$. By the mathematical induction, we know that (9) holds for all $t \geqslant t_{0}$. For any given $t \in\left[t_{m}, t_{m+1}\right)$, one can get

$$
\begin{aligned}
& \sum_{t_{k} \in\left(t_{0}, t\right]} \mathrm{e}^{-\lambda\left(t-t_{k}\right)} \chi_{2}\left(\left|u_{d}\left(t_{k}^{-}\right)\right|\right) \\
& \quad \leqslant \frac{1}{1-\mathrm{e}^{-\lambda \rho}} \chi_{2}\left(\max _{t_{k} \in\left(t_{0}, t\right]}\left\{\left|u_{d}\left(t_{k}^{-}\right)\right|\right\}\right) .
\end{aligned}
$$

It follows from (9), (31), and the definition of $J(t)$ that

$$
\begin{aligned}
\mathbb{E} V(t) \leqslant & \mathrm{e}^{-\lambda\left(t-t_{0}\right)} \alpha_{2}\left(\mathbb{E}\|\xi\|^{p}\right)+\frac{1}{c-c^{\prime}} \chi_{1}\left(\left\|u_{c}\right\|_{\infty}\right) \\
& +\frac{1}{1-\mathrm{e}^{-\lambda \rho}} \chi_{2}\left(\max _{t_{k} \in\left(t_{0}, t\right]}\left\{\left|u_{d}\left(t_{k}^{-}\right)\right|\right\}\right) .
\end{aligned}
$$

By casualty,

$$
\begin{aligned}
\mathbb{E} V(t) \leqslant & \mathrm{e}^{-\lambda\left(t-t_{0}\right)} \alpha_{2}\left(\mathbb{E}\|\xi\|^{p}\right)+\frac{1}{c-c^{\prime}} \chi_{1}\left(\left\|u_{c}\right\|_{\left[t_{0}, t\right]}\right) \\
& +\frac{1}{1-\mathrm{e}^{-\lambda \rho}} \chi_{2}\left(\max _{t_{k} \in\left(t_{0}, t\right]}\left\{\left|u_{d}\left(t_{k}^{-}\right)\right|\right\}\right) .
\end{aligned}
$$

Then by condition (i) and Jensen's inequality, the required assertion (2) holds with $\beta(r, s)=\mathrm{e}^{-\lambda s} \alpha_{2}(r), \gamma_{c}(r)=(1 /(c-$ $\left.\left.c^{\prime}\right)\right) \chi_{1}(r)$ and $\gamma_{d}(r)=\left(1 /\left(1-\mathrm{e}^{-\lambda \rho}\right)\right) \chi_{2}(r)$. By Lemma 4.2 in [31], it is easy to see that $\beta \in \mathscr{K} \mathscr{L}, \gamma_{c}, \gamma_{d} \in \mathscr{K}_{\infty}$. As $\beta, \gamma_{c}, \gamma_{d}$ are independent of the particular choice of the impulse time sequence, system (1) is uniformly $p$-ISS over $\delta_{\min }(\rho)$.

For the special case $\mu=1, \mu \mathrm{e}^{-c \rho}<1$ holds for any $\rho>0$, so system (1) is uniformly $p$-ISS over $\mathcal{S}_{\min }(\rho)$ for any $\rho>0$. In other words, system (1) is uniformly $p$-ISS over $\mathcal{S}_{\text {all }}$. The proof is complete.

Remark 9. When $\mu>1$, condition (iii) implies that the impulses may be destabilizing. So, in order to maintain the p-ISS property of system (1), the impulse interval is required to be large enough to reduce the effect of the impulses. When $\mu=1$, the discrete dynamics are marginally stable for the zero input. In this case, the $p$-ISS of system (1) is not affected by the impulses.

With minor modification to the conditions of Theorem 8 , a criterion on SISS can be obtained as follows.

Theorem 10. Assume that conditions (ii) and (iii) of Theorem 8 hold, while condition (i) is replaced by

$$
\left(i^{*}\right) \alpha_{1}(|x|) \leqslant V(t, x) \leqslant \alpha_{2}(|x|),
$$

where $\alpha_{1}, \alpha_{2} \in \mathscr{K}_{\infty}$. Then, for any given $\rho>0$ satisfying $\mu \mathrm{e}^{-c \rho}<1$, system (1) is uniformly SISS over $\mathcal{S}_{\min }(\rho)$. In particular, when $\mu=1$, system (1) is uniformly SISS over $\mathcal{S}_{\text {all }}$.

Proof. By condition $\left(\mathrm{i}^{*}\right),(10)$ can be replaced by

$$
\begin{array}{r}
J(t)=\mathbb{E} V(t) \mathrm{e}^{\lambda\left(t-t_{0}\right)} \leqslant \mathbb{E} \alpha_{2}(|x(t)|) \leqslant \mathbb{E} \alpha_{2}(\|\xi\|), \\
t \in\left[t_{0}-\tau, t_{0}\right] .
\end{array}
$$

Then, following the same lines of the proof of Theorem 8 , it is easy to see that

$$
\begin{aligned}
\mathbb{E} V(t) \leqslant & \mathrm{e}^{-\lambda\left(t-t_{0}\right)} \mathbb{E} \alpha_{2}(\|\xi\|)+\frac{1}{c-c^{\prime}} \chi_{1}\left(\left\|u_{c}\right\|_{\left[t_{0}, t\right]}\right) \\
& +\frac{1}{1-\mathrm{e}^{-\lambda \rho}} \chi_{2}\left(\max _{t_{k} \in\left(t_{0}, t\right]}\left\{\left|u_{d}\left(t_{k}^{-}\right)\right|\right\}\right)
\end{aligned}
$$

holds for all $t \geqslant t_{0}$. Consequently, by Chebyshev's inequality, it follows that

$$
\begin{aligned}
& P\left\{V(t)-\mathrm{e}^{-\lambda\left(t-t_{0}\right)} \alpha_{2}(\|\xi\|)\right. \\
& \geqslant \delta\left(\frac{1}{c-c^{\prime}} \chi_{1}\left(\left\|u_{c}\right\|_{\left[t_{0}, t\right]}\right)\right. \\
& \left.\left.\quad+\frac{1}{1-\mathrm{e}^{-\lambda \rho}} \chi_{2}\left(\max _{t_{k} \in\left(t_{0}, t\right]}\left\{\left|u_{d}\left(t_{k}^{-}\right)\right|\right\}\right)\right)\right\} \\
& \leqslant \mathbb{E} V(t)-\mathbb{E} \alpha_{2}(\|\xi\|) \mathrm{e}^{-\lambda\left(t-t_{0}\right)} \\
& \quad \times\left(\frac{1}{c-c^{\prime}} \chi_{1}\left(\left\|u_{c}\right\|_{\left[t_{0}, t\right]}\right)\right. \\
& \left.\left.\quad+\frac{1}{1-\mathrm{e}^{-\lambda \rho}} \chi_{2}\left(\max _{t_{k} \in\left(t_{0}, t\right]}\left\{\left|u_{d}\left(t_{k}^{-}\right)\right|\right\}\right)\right)\right)^{-1}
\end{aligned}
$$

$\leqslant \varepsilon$,

where $\varepsilon$ can be made arbitrarily small by an appropriate choice of $\delta \in \mathscr{K}_{\infty}$. That is,

$$
\begin{aligned}
& P\left\{V(t)<\mathrm{e}^{-\lambda\left(t-t_{0}\right)} \alpha_{2}(\|\xi\|)\right. \\
& +\delta\left(\frac{1}{c-c^{\prime}} \chi_{1}\left(\left\|u_{c}\right\|_{\left[t_{0}, t\right]}\right)\right. \\
& \left.\left.\quad+\frac{1}{1-\mathrm{e}^{-\lambda \rho}} \chi_{2}\left(\max _{t_{k} \in\left(t_{0}, t\right]}\left\{\left|u_{d}\left(t_{k}^{-}\right)\right|\right\}\right)\right)\right\} \\
& >1-\varepsilon
\end{aligned}
$$

which yields

$$
\begin{gathered}
P\left\{V(t)<\beta\left(\|\xi\|, t-t_{0}\right)+\gamma_{c}\left(\left\|u_{c}\right\|_{\left[t_{0}, t\right]}\right)\right. \\
\left.\quad+\gamma_{d}\left(\max _{t_{k} \in\left(t_{0}, t\right]}\left\{\left|u_{d}\left(t_{k}^{-}\right)\right|\right\}\right)\right\}>1-\varepsilon,
\end{gathered}
$$

where $\beta(r, s)=\mathrm{e}^{-\lambda s} \alpha_{2}(r), \gamma_{c}(r)=\delta\left(\left(2 /\left(c-c^{\prime}\right)\right) \chi_{1}(r)\right), \gamma_{d}(r)=$ $\delta\left(\left(2 /\left(1-\mathrm{e}^{-\lambda \rho}\right)\right) \chi_{2}(r)\right)$. By condition $\left(\mathrm{i}^{*}\right)$, we know that $(3)$ holds. Therefore, system (1) is uniformly SISS over $\mathcal{S}_{\min }(\rho)$ and the proof is complete.

In view of Definitions 1-5, it is easy to obtain the following criteria on $p$-GAS and GASiP according to Theorems 8 and 10 . 
Corollary 11. Assume that there exist functions $V \in v_{0}, \alpha_{1} \in$ $c \mathscr{K}_{\infty}, \alpha_{2} \in v \mathscr{K}_{\infty}$ and scalars $q>1, c>0, \mu \in[1, q)$ such that

(i) $\alpha_{1}\left(|x|^{p}\right) \leqslant V(t, x) \leqslant \alpha_{2}\left(|x|^{p}\right)$;

(ii) $\mathbb{E} \mathscr{L} V(t, \varphi) \leqslant-c \mathbb{E} V(t, \varphi(0))$, for all $t \geqslant t_{0}, t \neq t_{k}$ and $\varphi \in P C_{\mathscr{F}_{t}}\left([-\tau, 0] ; \mathbb{R}^{n}\right)$ whenever $\mathbb{E} V(t+\theta, \varphi(\theta)) \leqslant$ $q \mathbb{E} V(t, \varphi(0)) ;$

(iii) $\mathbb{E} V\left(t_{k}, I_{k}\left(t_{k}^{-}, x, u_{d}\right)\right) \leqslant \mu \mathbb{E} V\left(t_{k}, x\right)$.

Then, for any given $\rho>0$ satisfying $\mu \mathrm{e}^{-c \rho}$, system (1) is uniformly $p$-GAS over $\mathcal{S}_{\min }(\rho)$. In particular, when $\mu=1$, system (1) is uniformly $p$-GAS over $\mathcal{S}_{\text {all }}$.

Corollary 12. Assume that conditions (ii) and (iii) of Corollary 11 hold, while condition (i) is replaced by

$$
\left(i^{*}\right) \alpha_{1}(|x|) \leqslant V(t, x) \leqslant \alpha_{2}(|x|),
$$

where $\alpha_{1}, \alpha_{2} \in \mathscr{K}_{\infty}$. Then, for any given $\rho>0$ satisfying $\mu \mathrm{e}^{-c \rho}<1$, system (1) is uniformly GASiP over $\mathcal{S}_{\min }(\rho)$. In particular, when $\mu=1$, system (1) is uniformly GASiP over $\mathcal{S}_{\text {all }}$.

Now let us apply the obtained results to the linear impulsive stochastic delayed system with the following form:

$$
\begin{aligned}
\mathrm{d} x= & \left(A x(t)+A_{1} x_{\tau}+B u_{c}(t)\right) \mathrm{d} t \\
& +\left(C x(t)+C_{1} x_{\tau}+D u_{c}(t)\right) \mathrm{d} w, \quad t \neq t_{k}, \\
x & \left(t_{k}\right)=E x\left(t_{k}^{-}\right)+F u_{d}\left(t_{k}^{-}\right), \quad k \in \mathbb{N},
\end{aligned}
$$

on $t \geqslant t_{0}$ with initial data $x_{t_{0}}=\xi$, where $x \in \mathbb{R}^{n}$ and $u_{c} \in$ $\mathscr{L}_{\infty}^{m_{1}}, u_{d} \in \mathscr{L}_{\infty}^{m_{2}}$ are system state and inputs, respectively; $x_{\tau}$ is short for $x(t-\tau) ; A, A_{1}, B, C, C_{1}, D, E, F$ are constant matrices with appropriate dimensions.

Corollary 13. Assume that there exist a matrix $P>0$ and constants $\lambda_{1}<0, \lambda_{2}>0, \lambda_{3}>0, \lambda_{4}>1, \lambda_{5}>0$ satisfying $\lambda_{4} \mathrm{e}^{\left(\lambda_{1}+\lambda_{2} p\right) \rho}<1$ such that the following matrix inequalities hold:

$$
\begin{gathered}
{\left[\begin{array}{ccc}
A^{T} P+P A+C^{T} P C-\lambda_{1} P & P A_{1}+C^{T} P C_{1} & P B+C^{T} P D \\
* & C_{1}^{T} P C_{1}-\lambda_{2} P & C_{1}^{T} P D \\
* & * & D^{T} P D-\lambda_{3} I
\end{array}\right]} \\
\leqslant 0, \\
\end{gathered}
$$

Then system (39) is uniformly ISS in mean square and uniformly SISS over $\mathcal{S}_{\min }(\rho)$.

Proof. We choose the candidate ISS-Lyapunov function $V(t, x)=x^{\mathrm{T}} P x$. By using $(40)$, and in view of the fact that $\lambda_{\text {min }}(P)|x|^{2} \leqslant x^{\mathrm{T}} P x \leqslant \lambda_{\text {max }}(P)|x|^{2}$, we can obtain by simple calculation that

$$
\begin{aligned}
\mathscr{L} V(t, x, u) & \left(\begin{array}{c}
x \\
x_{\tau} \\
u_{c}
\end{array}\right) \\
& \times\left(\begin{array}{ccc}
A^{\mathrm{T}} P+P A+C^{\mathrm{T}} P C & P A_{1}+C^{\mathrm{T}} P C_{1} & P B+C^{\mathrm{T}} P D \\
* & C_{1}^{\mathrm{T}} P C_{1} & C_{1}^{\mathrm{T}} P D \\
* & * & D^{\mathrm{T}} P D
\end{array}\right) \\
& \times\left(\begin{array}{c}
x \\
x_{\tau} \\
u_{c}
\end{array}\right) \quad \lambda_{1} x^{\mathrm{T}} P x+\lambda_{2} x_{\tau}^{\mathrm{T}} P x_{\tau}+\lambda_{3} u_{c}^{\mathrm{T}} u_{c} .
\end{aligned}
$$

So, whenever $\mathbb{E} V(t+\theta) \leqslant q \mathbb{E} V(t)$, we have

$$
\mathbb{E} \mathscr{L} V(t, x, u) \leqslant\left(\lambda_{1}+\lambda_{2} q\right) \mathbb{E} V(t, x)+\lambda_{3}\left|u_{c}\right|^{2}
$$

On the other hand,

$$
\begin{aligned}
V\left(t_{k}, x\left(t_{k}\right)\right) & =\left(\begin{array}{c}
x \\
u_{d}
\end{array}\right)^{\mathrm{T}}\left(\begin{array}{cc}
E^{\mathrm{T}} P E & E^{\mathrm{T}} P F \\
* & F^{\mathrm{T}} P F
\end{array}\right)\left(\begin{array}{c}
x \\
u_{d}
\end{array}\right) \\
& \leqslant \lambda_{4} x^{\mathrm{T}} P x+\lambda_{5} u_{d}^{\mathrm{T}} u_{d} .
\end{aligned}
$$

So,

$$
\mathbb{E} V\left(t_{k}, x\left(t_{k}\right)\right) \leqslant \lambda_{4} \mathbb{E} V\left(t_{k}^{-}\right)+\lambda_{5}\left|u_{d}\right|^{2}
$$

It is obvious that all conditions of Theorem 8 are satisfied, with $c=-\left(\lambda_{1}+\lambda_{2} p\right)$ and $\mu=\lambda_{4}$. Therefore, we conclude by Theorems 8 and 10 that system (39) is uniformly $p$-ISS and uniformly SISS over $\mathcal{S}_{\min }(\rho)$.

Remark 14. It is noted that (40) are not linear with the combined variables $\left(P, \lambda_{1}, \lambda_{2}, \lambda_{4}\right)$, and, therefore, they are not linear matrix inequalities (LMIs). This makes the computation difficult but also flexible. We can first assign $\lambda_{1}, \lambda_{2}$, and $\lambda_{4}$ and then solve LMIs (40) by using the Matlab LMI Toolbox.

\section{Illustrative Example}

In this section, to illustrate the validity of our results, we give the following linear numerical example. We point out that, due to the effect of the input $u$, the state $x(t)$ will not converge to zero but will remain bounded (in the sense of mean square or in probability), which is consistent with the definition of p-ISS/SISS. 


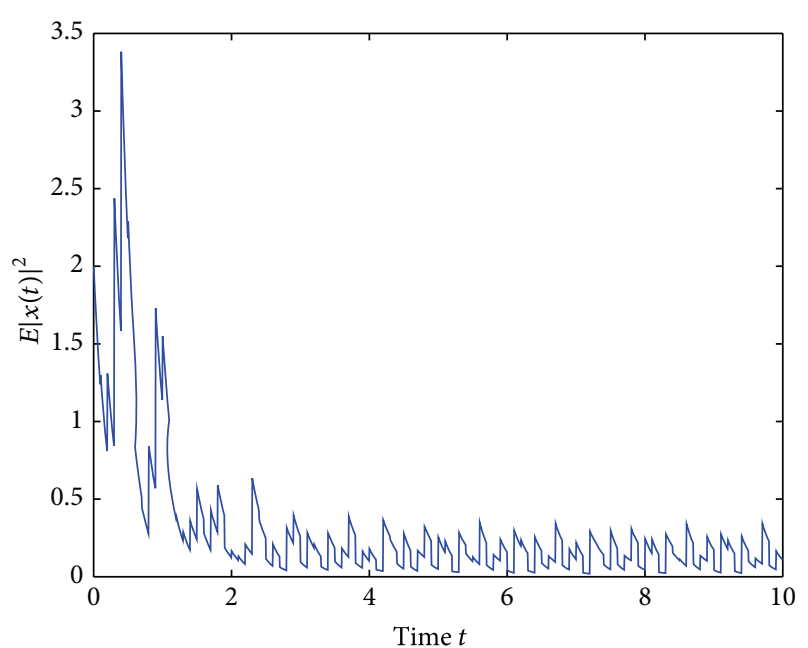

FIGURE 1: The mean square of the solution with external input (2000 samples).

Example 1. Consider system (39) with the following parameters:

$$
\begin{gathered}
A=\operatorname{diag}(-3,-2.5), \quad A_{1}=\left[\begin{array}{ll}
0.3 & 0.1 \\
0.1 & 0.2
\end{array}\right], \quad B=\left[\begin{array}{l}
0.1 \\
0.1
\end{array}\right], \\
C=\left[\begin{array}{ll}
0.2 & 0.4 \\
0.3 & 0.1
\end{array}\right], \quad C_{1}=\left[\begin{array}{cc}
-0.2 & 0.1 \\
0 & 0.3
\end{array}\right], \quad D=\left[\begin{array}{l}
0.1 \\
0.1
\end{array}\right], \\
E=\left[\begin{array}{cc}
1.1 & 0.2 \\
-0.3 & 1.2
\end{array}\right], \quad F=\left[\begin{array}{l}
0.2 \\
0.5
\end{array}\right] .
\end{gathered}
$$

Setting $\lambda_{1}=-3.8, \lambda_{2}=0.2, \lambda_{4}=1.4$, and solving LMIs (40) by using the Matlab LMI Toolbox, then

$$
\begin{gathered}
P=\left[\begin{array}{cc}
395.5597 & -72.7854 \\
395.5597 & 269.6470
\end{array}\right], \quad \lambda_{3}=0.9546 \\
\lambda_{5}=3.5515
\end{gathered}
$$

is a group of feasible solutions. Choosing $p=1.41>\mu=$ $\lambda_{4}=1.4, \rho=0.1$, it is easy to check that all the conditions of Corollary 13 are satisfied, which means that the system is uniform ISS in mean square and uniform SISS for arbitrary sequence of impulse times satisfying $\inf \left\{t_{k}-t_{k-1}\right\} \geqslant 0.1$. The sample path and the mean square of the solution are displayed in Figures 1 and 2, respectively, where $\tau=0.5$, initial data $\xi(\theta)=\left[\begin{array}{ll}1 & -1\end{array}\right]^{\mathrm{T}}$ for $\theta \in[-0.5,0]$, and impulse interval $t_{k}-$ $t_{k-1}=0.1$ and external inputs $u_{c}(t)=u_{d}(t)=\sin t$.

As $p$-ISS/SISS implies $p$-GAS/GASiP of the corresponding unforced system, we conclude that the system with $u_{c}=$ $u_{d} \equiv 0$ is uniform GAS in mean square and GASiP for arbitrary sequence of impulse times satisfying inf $\left\{t_{k}-t_{k-1}\right\} \geqslant$ 0.1 . The simulations of the unforced system are shown in Figures 3 and 4.

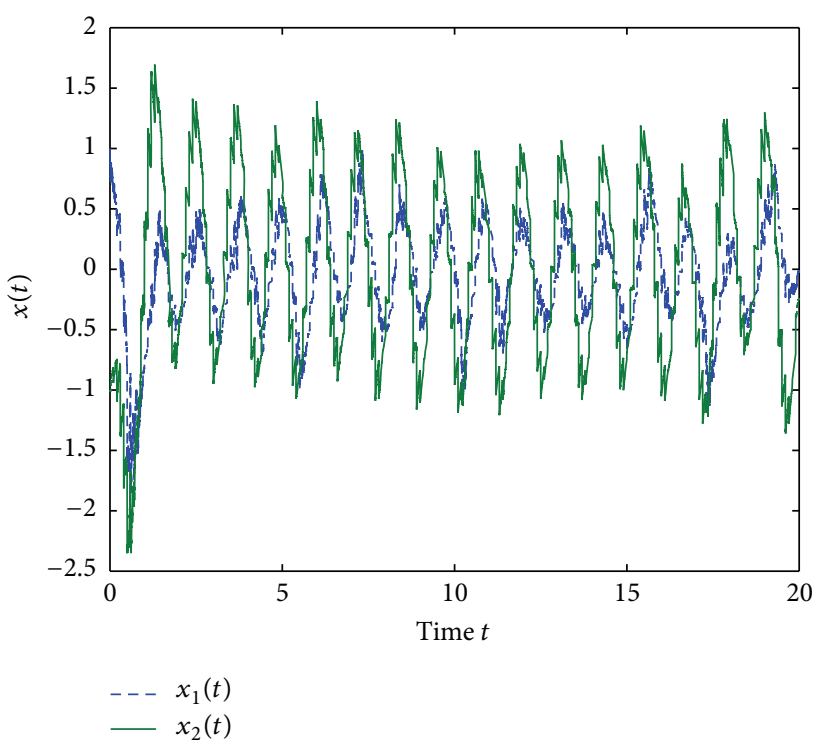

FIGURE 2: The state trajectory of the system with external input (single sample).

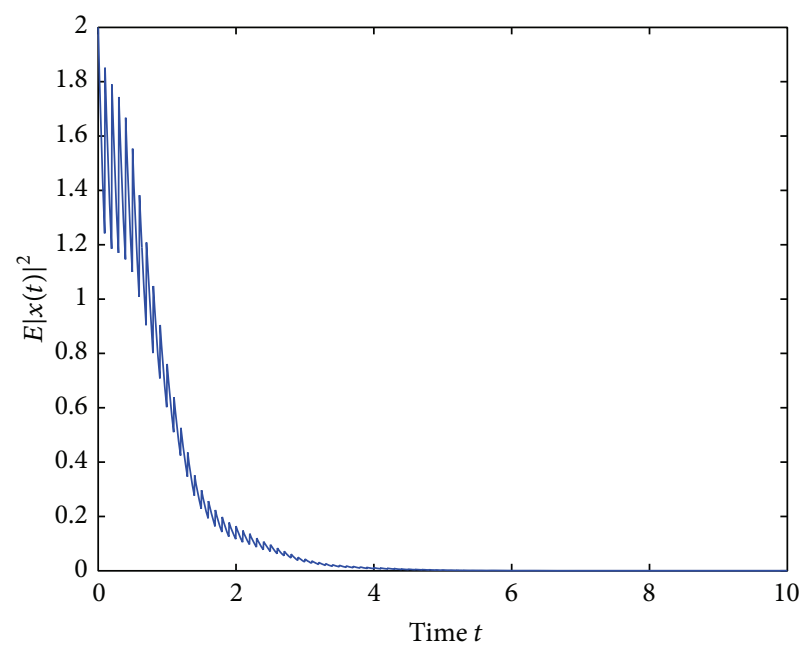

FIgURE 3: The mean square of the solution without external input (2000 samples).

\section{Conclusions}

This paper has investigated the $p$-ISS/SISS of impulsive stochastic systems with external inputs. By combining stochastic analysis techniques, piecewise continuous Lyapunov functions, and Razumikhin techniques, sufficient conditions for uniform $p$-ISS/SISS over a given class of impulse times sequences have been established. As a byproduct, the criteria on $p$-GAS/GASiP are also derived. For future research, interesting topics may include establishing p-ISS/SISS theorems with stabilizing impulses, as well as p-ISS/SISS analysis by exploring new techniques such as Lyapunov-Krosovskii functional method. 


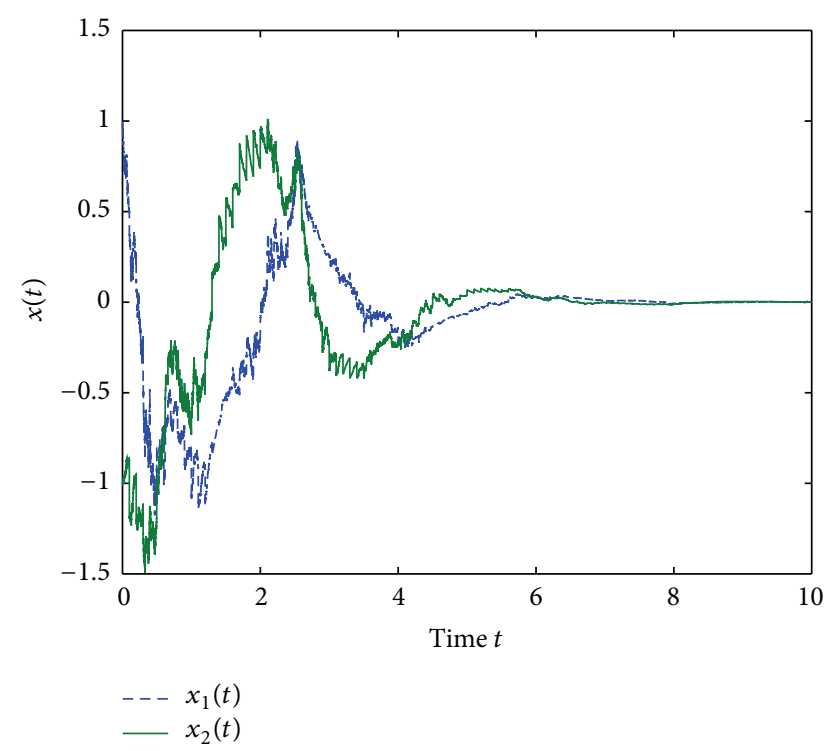

FIGURE 4: The state trajectory of the system without external input (single sample).

\section{Conflict of Interests}

The authors declare that there is no conflict of interests regarding the publication of this paper.

\section{Acknowledgments}

This work was supported by the Excellent Youthful Talent Foundation of Colleges and Universities of Anhui Province of China (2013SQRL024ZD), the Research Foundation for Young Scientists of Anhui University of Technology (QZ201314), the National Natural Science Foundation of China (11301004), and Anhui Provincial Nature Science Foundation (1308085QA15).

\section{References}

[1] P. Zhao, W. Feng, and Y. Kang, "Stochastic input-to-state stability of switched stochastic nonlinear systems," Automatica, vol. 48, pp. 2569-2576, 2012.

[2] E. D. Sontag, "Smooth stabilization implies coprime factorization," IEEE Transactions on Automatic Control, vol. 34, no. 4, pp. 435-443, 1989.

[3] Z.-P. Jiang, A. R. Teel, and L. Praly, "Small-gain theorem for ISS systems and applications," Mathematics of Control, Signals, and Systems, vol. 7, no. 2, pp. 95-120, 1994.

[4] L. Praly and Z.-P. Jiang, "Stabilization by output feedback for systems with ISS inverse dynamics," Systems and Control Letters, vol. 21, no. 1, pp. 19-33, 1993.

[5] L. Praly and Y. Wang, "Stabilization in spite of matched unmodeled dynamics and an equivalent definition of input-to-state stability," Mathematics of Control, Signals, and Systems, vol. 9, no. 1, pp. 1-33, 1996.

[6] C. Cai and A. R. Teel, "Input-output-to-state stability for discrete-time systems," Automatica, vol. 44, no. 2, pp. 326-336, 2008.
[7] M. Lazar, W. P. M. H. Heemels, and A. R. Teel, "Further inputto-state stability subtleties for discrete-time systems," IEEE Transactions on Automatic Control, vol. 58, pp. 1609-1613, 2013.

[8] J. Liu, X. Liu, and W.-C. Xie, "Input-to-state stability of impulsive and switching hybrid systems with time-delay," Automatica, vol. 47, no. 5, pp. 899-908, 2011.

[9] M. A. Mueller and D. Liberzon, "Input/output-to-state stability and state-norm estimators for switched nonlinear systems," Automatica, vol. 48, pp. 2029-2039, 2012.

[10] F. L. Sun, Z. H. Guan, X. H. Zhang, and J. C. Chen, "Exponential-weighted input-to-state stability of hybrid impulsive switched systems," IET Control Theory and Applications, vol. 6, pp. 430-436, 2012.

[11] Y. E. Wang, R. Wang, and J. Zhao, "Input-to-state stability of non-linear impulsive and switched delay systems," IET Control Theory and Applications, vol. 7, pp. 1179-1185, 2013.

[12] W.-H. Chen and W. X. Zheng, "Input-to-state stability for networked control systems via an improved impulsive system approach," Automatica, vol. 47, no. 4, pp. 789-796, 2011.

[13] Z. Liu, R. E. Torres, N. Patel, and Q. Wang, "Further development of input-to-state stabilizing control for dynamic neural network systems," IEEE Transactions on Systems, Man, and Cybernetics Part A: Systems and Humans, vol. 38, no. 6, pp. 14251433, 2008.

[14] E. N. Sanchez and J. P. Perez, "Input-to-state stabilization of dynamic neural networks," IEEE Transactions on Systems, Man, and Cybernetics Part A: Systems and Humans, vol. 33, no. 4, pp. 532-536, 2003.

[15] S. Zhu and Y. Shen, "Two algebraic criteria for input-to-state stability of recurrent neural networks with time-varying delays," Neural Computing and Applications, vol. 22, pp. 1163-1169, 2013.

[16] V. Lakshmikantham, D. D. Bainov, and P. S. Simenov, Theory of Impulsive Differential Equations, World Scientific, Singapore, 1989.

[17] J. P. Hespanha, D. Liberzon, and A. R. Teel, "Lyapunov conditions for input-to-state stability of impulsive systems," Automatica, vol. 44, no. 11, pp. 2735-2744, 2008.

[18] W.-H. Chen and W. X. Zheng, "Input-to-state stability and integral input-to-state stability of nonlinear impulsive systems with delays," Automatica, vol. 45, no. 6, pp. 1481-1488, 2009.

[19] S. Dashkovskiy, M. Kosmykov, A. Mironchenko, and L. Naujok, "Stability of interconnected impulsive systems with and without time delays, using Lyapunov methods," Nonlinear Analysis: Hybrid Systems, vol. 6, no. 3, pp. 899-915, 2012.

[20] X. Mao, Stochastic Differential Equations and Applications, Horwood, Chichester, UK, 2nd edition, 2007.

[21] Z. Wu, P. Shi, H. Su, and J. Chu, "Asynchronous $l_{2}-l_{\infty}$ filtering for discrete-time stochastic markov jump systems with randomly occurred sensor nonlinearities," Automatica, vol. 50, pp. 180-186, 2014.

[22] Z.-G. Wu, P. Shi, H. Su, and J. Chu, "Passivity analysis for discrete-time stochastic markovian jump neural networks with mixed time delays," IEEE Transactions on Neural Networks, vol. 22, no. 10, pp. 1566-1575, 2011.

[23] H. Shen, S. Xu, J. Lu, and J. Zhou, "Passivity-based control for uncertain stochastic jumping systems with mode-dependent round-trip time delays," Journal of the Franklin Institute, vol. 349, no. 5, pp. 1665-1680, 2012.

[24] S. Peng and Y. Zhang, "Razumikhin-type theorems on pth moment exponential stability of impulsive stochastic delay differential equations," IEEE Transactions on Automatic Control, vol. 55, no. 8, pp. 1917-1922, 2010. 
[25] L. Xu and D. Xu, "Mean square exponential stability of impulsive control stochastic systems with time-varying delay," Physics Letters, Section A: General, Atomic and Solid State Physics, vol. 373, no. 3, pp. 328-333, 2009.

[26] F. Yao and F. Deng, "Stability of impulsive stochastic functional differential systems in terms of two measures via comparison approach," Science China Information Sciences, vol. 55, no. 6, pp. 1313-1322, 2012.

[27] F. Yao and F. Deng, "Exponential stability in terms of two measures of impulsive stochastic functional differential systems via comparison principle," Statistics and Probability Letters, vol. 82, no. 6, pp. 1151-1159, 2012.

[28] F. Yao, F. Deng, and P. Cheng, "Exponential stability of impulsive stochastic functional differential systems with delayed impulses," Abstract and Applied Analysis, vol. 2013, Article ID 548712, 8 pages, 2013.

[29] S.-J. Liu, J.-F. Zhang, and Z.-P. Jiang, "A notion of stochastic input-to-state stability and its application to stability of cascaded stochastic nonlinear systems," Acta Mathematicae Applicatae Sinica, vol. 24, no. 1, pp. 141-156, 2008.

[30] L. Huang and X. Mao, "On input-to-state stability of stochastic retarded systems with Markovian switching," IEEE Transactions on Automatic Control, vol. 54, no. 8, pp. 1898-1902, 2009.

[31] H. Khalil, Nonlinear Systems, Prentice Hall, New Jersey, NJ, USA, 3rd edition, Edition, 2002. 


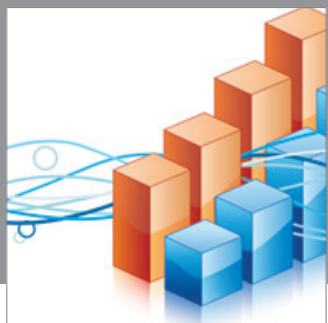

Advances in

Operations Research

mansans

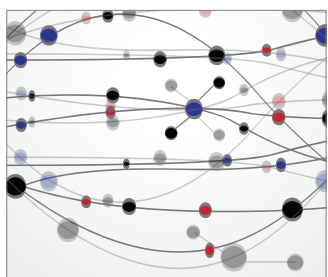

The Scientific World Journal
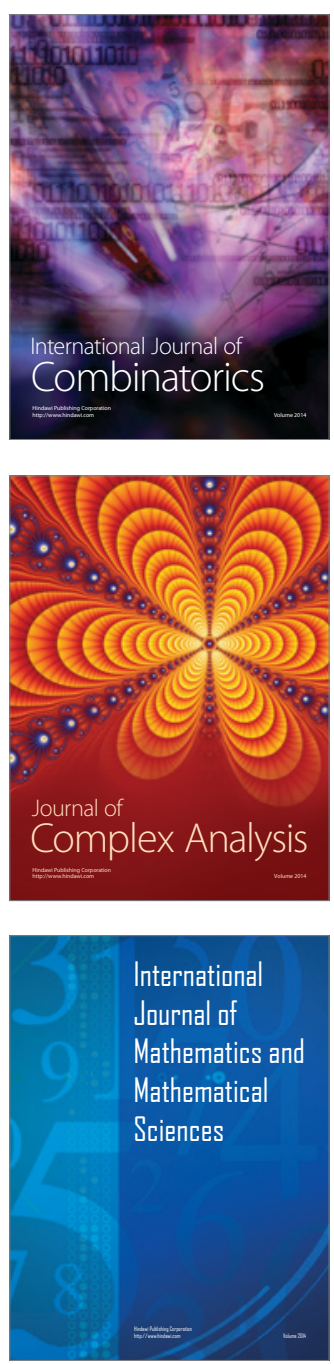
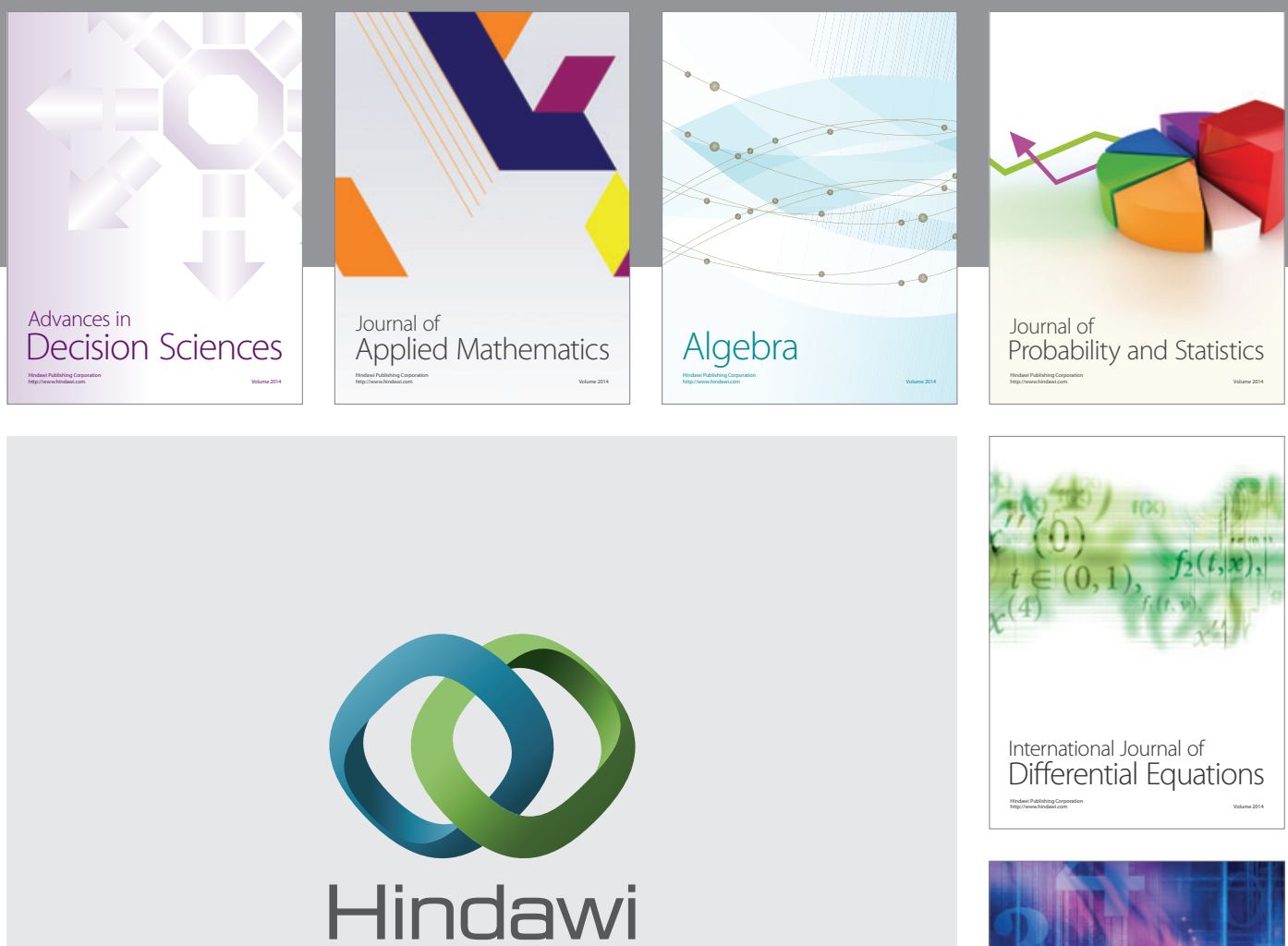

Submit your manuscripts at http://www.hindawi.com
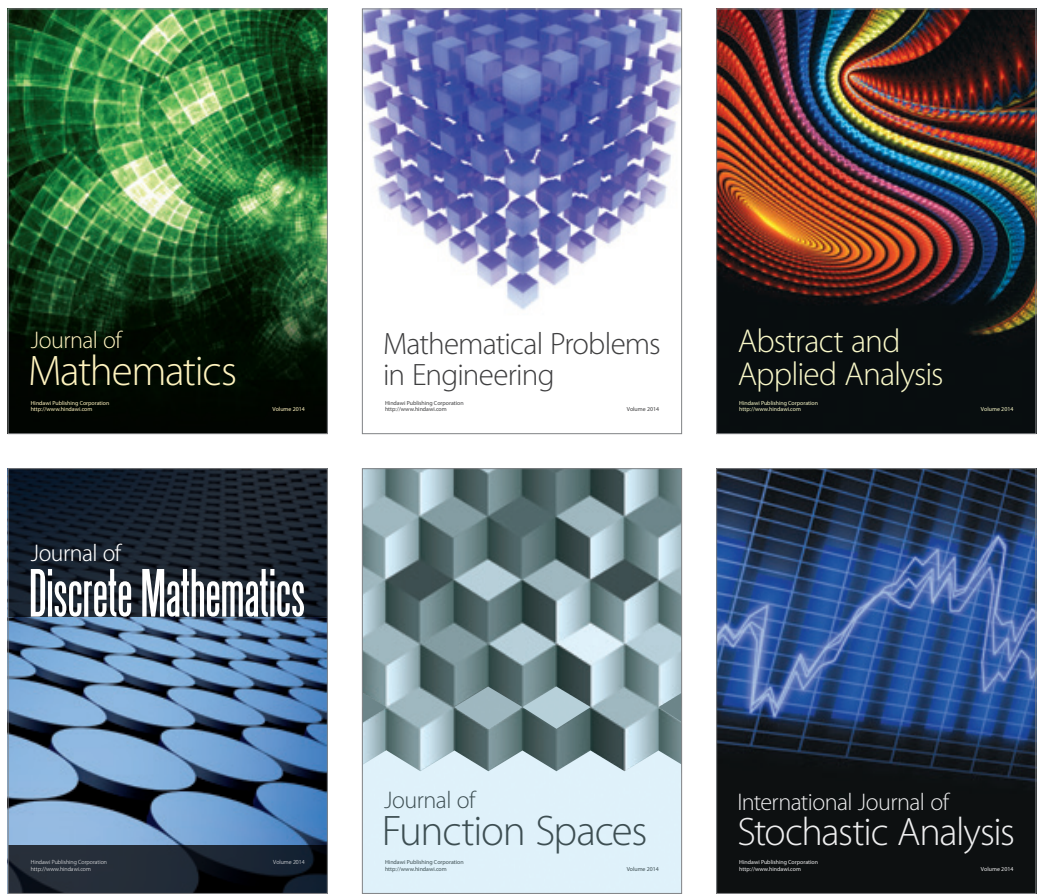

Journal of

Function Spaces

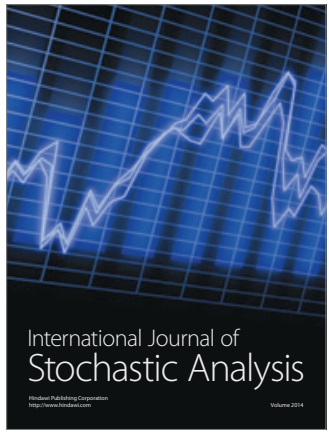

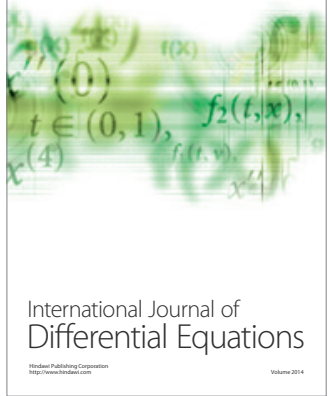
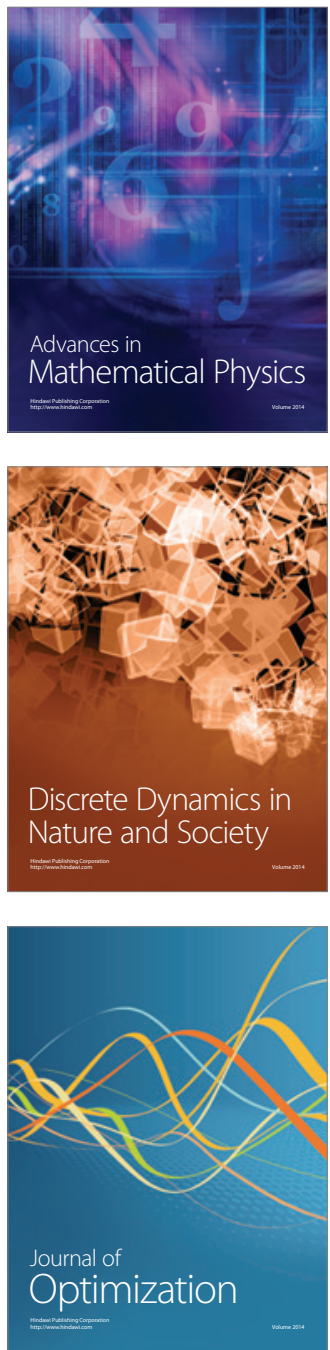